\title{
Recurrence risk after first febrile seizure and effect of short term diazepam prophylaxis
}

\author{
F URSIN KNUDSEN \\ Department of Paediatrics, Glostrup University Hospital, Denmark
}

SUMMARY In a prospective randomised study, 289 children admitted consecutively to hospital with their first febrile seizure were allocated, by date of admission, to short term diazepam prophylaxis $(n=152)$ or to no prophylaxis $(n=137)$ and followed for 18 months. In untreated children, five major risk factors for recurrent febrile convulsions were identified: age 15 months or less at the time of the first febrile seizure, epilepsy in first degree relatives, febrile convulsions in first degree relatives, a first complex febrile seizure, and day nursery care. The 18 month recurrence rate was 80 to $100 \%$ if three to five risk factors were present, $50 \%$ if two factors were identified, $25 \%$ where one factor was found, and $12 \%$ if there were no predictors. During prophylaxis the recurrence rate was uniformly low (mean $12 \%$ ) in all risk groups. In high (three or more factors) and intermediate (two factors) risk children prophylaxis provided effective seizure control and reduced the recurrence rate from $80 \%$, or more, to $12 \%$ and $50 \%$ to $12 \%$, respectively. In children with one risk factor $50 \%$ of all recurrences were prevented $(25 \%$ to $12 \%)$. Prophylaxis was ineffective in very low risk children $(12 \%$ to $12 \%)$.

In recent studies various risk factors for recurrent febrile convulsions have been identified..$^{1-4}$ There is, however, little available evidence on the interaction of these risk factors and on their influence on the recurrence risk with or without prophylaxis.

In a prospective randomised study we investigated the efficacy of short term diazepam prophylaxis in 289 children referred consecutively to hospital with their first febrile seizure. The results from this study have already been reported. ${ }^{5-6}$ We have now identified major risk factors for recurrent febrile seizures in these 289 children, stratified the children according to the risk of a recurrence, and analysed the efficacy of prophylaxis in high, intermediate, and low risk children given either short term diazepam prophylaxis at times of fever for 18 months or no prophylaxis. Pharmacokinetic and clinical data on short term diazepam prophylaxis have also been reported elsewhere. ${ }^{7-8}$

\section{Material and methods}

Among 326 children referred consecutively to hospital during the period 1 June 1978 to 1 June 1980 with their first simple or complex febrile seizure, 289 were included in the study as they lived in our referral area of Copenhagen. Exclusion criteria were purulent meningitis, chronic cerebral disease, or a history of non-febrile seizures. Subject to their parents' informed consent the children were allocated by date of admission either to short term diazepam prophylaxis (even dates) for 18 months, that is rectal diazepam in solution (Apozepam, Apothekernes Laboratorium for Specialpræparater, Norway) $5 \mathrm{mg}$ ( 3 years, or less) to $7.5 \mathrm{mg}$ ( 3 or more years) at time of illness (when the temperature was $38.5^{\circ} \mathrm{C}$ or more) or to the control group (odd dates) given no prophylaxis and only rectal diazepam in case of further fits. In the prophylaxis group all parents were told to administer diazepam rectally whenever the child had a temperature of $38.5^{\circ} \mathrm{C}$ or more, and to repeat the treatment every 12 hours until the temperature fell below $38.5^{\circ} \mathrm{C}$. After finishing the present study we have changed our routine prophylaxis slightly. To avoid unnecessary sedation due to accumulation of diazepam a maximum of four consecutive doses are given per illness.

The patients were seen in the outpatient clinic, 3 , 6,12 , and 18 months after the first febrile seizure, and an electroencephalograph was obtained one month after the first febrile seizure. The total follow up period, with cut off at 18 months, was 2406 months in the prophylaxis group and 2093 months in the control group. A total of 29 children $(10 \%)$ were 
followed for 0 to 3 months, $19(6 \%)$ for 3 to 11 months, $45(16 \%)$ for 12 to 17 months and 196 $(68 \%)$ for 18 months or more.

The variables analysed simultaneously by Cox's multivariate step-wise regression analysis ${ }^{9}$ were: sex; age at the first febrile seizure; febrile convulsions in parents or siblings; epilepsy in parents or siblings; type of first febrile seizure, that is simple or complex (15 minutes or more, and/or focal and/or multiple, two or more fits per 24 hours); child care (home, day care, day nursery); and psychomotor development (normal, slightly retarded). The estimated recurrence rates were corrected for the varying follow up periods, that is to the values had all patients been followed throughout the 18 months' period. Only the first recurrence was taken into account in Cox's analysis.

The prophylaxis and control groups were stratified according to the number of risk factors identified in the individual child (0-5 items) into six groups, two low risk groups (none or one factor), an intermediate group (two factors), and three high risk groups (three, four, or five factors) (Table 1). Risk group 0 included children aged more than 15 months with simple febrile seizures without a family history of epilepsy or febrile convulsions, and cared for at home. Children in risk group 5 comprised all five factors: young age (15 months or less), a complex first febrile seizure, a positive family history of both epilepsy and febrile seizures, and day nursery care.

\section{Results}

Clinical data. The prophylaxis group $(n=152)$ consisted of 78 boys and 74 girls. A total of $140(93 \%)$ had had a short lasting (less than 15 minutes) and 12 (7\%) a long lasting (15 minutes or more) seizure. Some $130(86 \%)$ had had a simple and $22(14 \%)$ a complex first febrile seizure. A total of $60(40 \%)$ children were aged less than 15 months and 92 $(60 \%) 15$ months or more.

The control group $(n=137)$ (Table 1$)$ comprised 80 boys and 57 girls of whom $131(96 \%)$ had had convulsions of short duration and $6(4 \%)$ of long duration. Altogether $112(82 \%)$ had had a simple and $25(18 \%)$ a complex first seizure. No significant differences between the two groups were found in respect of sex, age, type and duration of convulsions, and family history of epilepsy and febrile convulsions.

The number of children with recurrences versus the total number of children in the stratified prophylaxis and control groups are given in Table 2 .

Table 1 Recurrence rate in relation to identified risk factors in children given no prophylaxis

\begin{tabular}{|c|c|c|c|c|c|c|c|}
\hline \multirow[t]{2}{*}{ Risk factor } & & \multirow{2}{*}{$\begin{array}{l}\text { Risk } \\
\text { score }\end{array}$} & \multirow{2}{*}{$\begin{array}{l}\text { No of } \\
\text { patients }\end{array}$} & \multicolumn{3}{|c|}{ Recurrence rate $(\%(S D))^{*}$} & \multirow[t]{2}{*}{$P^{*}$} \\
\hline & & & & 6 months & 12 months & 18 months & \\
\hline \multirow[t]{2}{*}{ Age: } & $<15$ month & 1 & 56 & $33(6)$ & $50(7)$ & $56(7)$ & \multirow{2}{*}{$<0.001$} \\
\hline & $\geqslant 15$ month & 0 & 81 & $15(4)$ & $18(5)$ & $23(5)$ & \\
\hline \multirow[t]{2}{*}{ Epilepsy in the family: } & Yes & 1 & 8 & 59 (17) & 59 (17) & 59 (17) & \multirow{2}{*}{$<0.01$} \\
\hline & No & 0 & 129 & $20(4)$ & $30(4)$ & $36(5)$ & \\
\hline \multirow{2}{*}{ Febrile convulsions in the family: } & Yes & 1 & 34 & $40(9)$ & $52(9)$ & $59(9)$ & \multirow{2}{*}{$=0.01$} \\
\hline & No & 0 & 103 & $12(4)$ & $27(5)$ & $30(5)$ & \\
\hline \multirow[t]{2}{*}{ Type of first febrile convulsions: } & Complex & 1 & 25 & $39(10)$ & $51(10)$ & $55(10)$ & \multirow{2}{*}{$<0.05$} \\
\hline & Simple & 0 & 112 & $20(4)$ & $28(5)$ & $33(5)$ & \\
\hline \multirow[t]{2}{*}{ Care: } & Day nursery & 1 & 46 & 37 (7) & $40(7)$ & $45(8)$ & \multirow{2}{*}{$=0.05$} \\
\hline & Home/day care & 0 & 91 & $21(6)$ & $30(5)$ & $37(9)$ & \\
\hline
\end{tabular}

${ }^{*} \mathrm{P}$ values and SD are derived from log rank test and Cox's analysis.

Table 2 Number of children with seizure recurrence within 18 months $v$ the total number of children, stratified according to the number of risk factors identified

\begin{tabular}{|c|c|c|c|c|c|}
\hline & \multicolumn{5}{|c|}{ No of risk factors } \\
\hline & 0 & 1 & 2 & 3 & $4-5$ \\
\hline \multicolumn{6}{|l|}{ Control group } \\
\hline Children with recurrences $(n=46)$ & $4(11 \%)$ & $14(26 \%)$ & $16(46 \%)$ & $9(82 \%)$ & $3(100 \%)$ \\
\hline Total no of children $(n=137)$ & 35 & 53 & 35 & 11 & 3 \\
\hline \multicolumn{6}{|l|}{ Prophylaxis group } \\
\hline Children with recurrences $(n=19)$ & $4(9 \%)$ & $11(17 \%)$ & $3(10 \%)$ & $1(13 \%)$ & 0 \\
\hline Total no of children $(n=152)$ & 46 & 66 & 31 & 8 & 1 \\
\hline Total no of children $(n=289)$ & $81(28 \%)$ & $118(40 \%)$ & $66(24 \%)$ & $19(7 \%)$ & $4(1 \%)$ \\
\hline
\end{tabular}


The age distribution did not differ significantly in the major risk groups $(\mathrm{P}=0 \cdot 1)$.

Risk factors in untreated children. Five major risk factors for subsequent febrile convulsions could be identified (Table 1). Early age at the time of the first febrile seizure was the variable most highly associated with recurrences. The age dependent risk was identified in many models, but was most noticeable by an age division at 15 months at the onset of the initial seizure.

In a model excluding the influence of the child's age, multivariate regression analysis identified four additional factors (Table 1), which per se significantly contributed to the recurrence risk: a history of epilepsy in first degree relatives, a history of febrile convulsions in first degree relatives, a first complex febrile seizure, and nursery care. Sex $(\mathrm{P}=0 \cdot 2)$ and psychomotor development $(\mathrm{P}=0 \cdot 2)$ did not significantly contribute to the recurrence risk.

Risk factors during prophylaxis. Prophylaxis eliminated all risk factors, except a first complex febrile seizure. Children with complex febrile seizure receiving diazepam at times of fever carried a high 18 months recurrence risk compared with children with simple febrile seizures $(55 \% \vee 27 \%, \mathrm{P}<0 \cdot 05){ }^{6}$

Assessment of recurrence risk. In the untreated group, high (three or more factors), intermediate (two factors), and low risk children (none or one factor) could be identified. The 18 month recurrence rate was 80 to $100 \%$ if three to five risk factors were

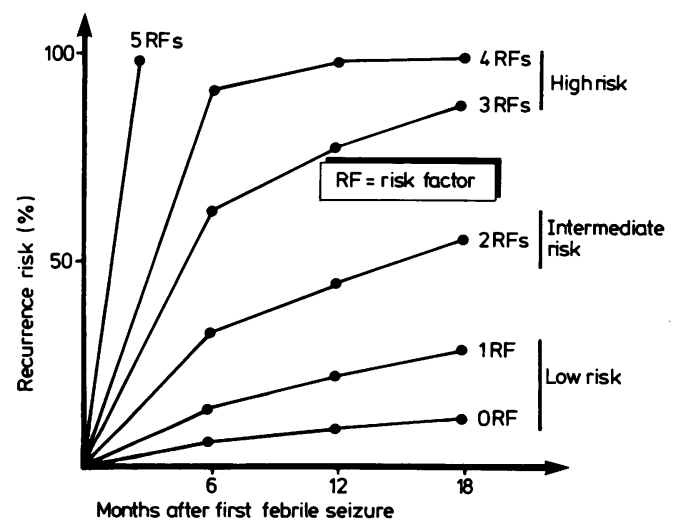

Fig. 1 Risk factors and recurrence risk without prophylaxis.

Without prophylaxis low risk (0-1 risk factor), intermediate risk ( 2 risk factors), and high risk children ( $\geqslant 3$ risk factors) could be identified. The recurrence rate is $12 \%$ in very low risk and 80 to $100 \%$ in high risk children after 18 months follow up. identified, $50 \%$ if two factors were present, $25 \%$ for one factor, and $12 \%$ in children without any risk factors (Fig. 1).

During prophylaxis the recurrence rate was uniformly low (mean $12 \%$ ) in all risk groups (Fig. 2).

The reduction in the recurrence risk associated with prophylaxis (Figs. 1 and 2) was strongly influenced by the number of predictors. In high and intermediate risk children, prophylaxis afforded effective seizure control and reduced the recurrence rate from $80 \%$ or more to $12 \%$ and from $50 \%$ to $12 \%$, respectively. In low risk children, comprising $40 \%, 50 \%$ of all the recurrences were prevented $(25 \%$ to $12 \%)$, whereas very low risk children $(28 \%$ of the study group) did not respond to prophylaxis $(12 \%$ to $12 \%)$.

The predictive value of the best positive or negative answer to the question of whether an individual child would have another febrile seizure within 18 months is given in Table 3.

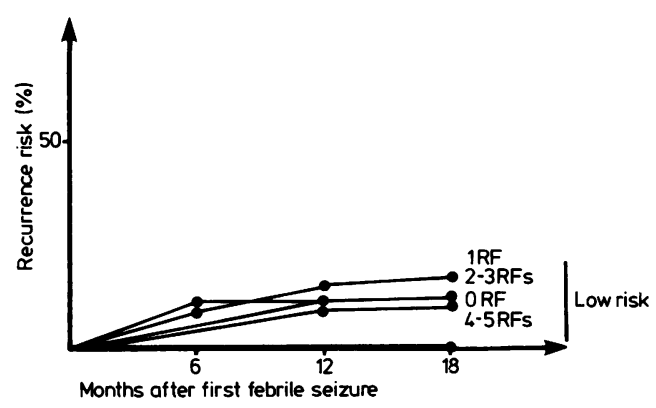

Fig. 2 Risk factors and recurrence risk during prophylaxis.

Prophylaxis with diazepam at times of fever eliminated the risk factors, except a first complex febrile convulsion (not shown). The recurrence rate is uniformly low. Prophylaxis is ineffective in very low risk children.

Table 3 Predictive value of the 18 months' recurrence risk assessment in the untreated child, who has experienced a first febrile seizure

\begin{tabular}{|c|c|c|c|c|}
\hline \multirow{2}{*}{$\begin{array}{l}\text { No of } \\
\text { risk } \\
\text { factors }\end{array}$} & \multicolumn{2}{|c|}{$\begin{array}{l}\text { Actual outcome } \\
\text { recurrence }(\%)\end{array}$} & \multirow{2}{*}{$\begin{array}{l}\text { Predicted outcome } \\
\text { recurrence, yes/no } \\
\text { best guess }\end{array}$} & \multirow{2}{*}{$\begin{array}{l}\text { Prediction } \\
\text { correct in } \\
\% \text { of cases }\end{array}$} \\
\hline & Yes & No & & \\
\hline 0 & 10 & 90 & no & 90 \\
\hline 1 & 25 & 75 & no & 75 \\
\hline 2 & 50 & 50 & yes/no & 50 \\
\hline 3 & 80 & 20 & yes & 80 \\
\hline $\left.\begin{array}{l}4 \\
5\end{array}\right\}$ & 100 & 0 & yes & 100 \\
\hline
\end{tabular}




\section{Discussion}

The study indicates that at least five factors influence the seizure recurrence rate. Furthermore, the number of predictors identified in each child seems to be useful in identifying those who are likely to have further febrile seizures. It seems that each risk factor, whether genetic or environmental, contributes to the recurrence risk in a cumulative way; the more items identified the higher the recurrence rate.

In an effort to estimate the recurrence risk more accurately, a prognostic index based on a six point scale was established. For practical purposes weak and strong predictors were assigned equal predictive power. In terms of risk groups, the risk assessment seemed to be reliable and a fairly good correlation between predicted and actual outcome was obtained. Thus, populations at low, intermediate, and high risk of future febrile seizures could readily be identified by means of the proposed scoring system. There is no reason, however, to suggest that the occurrence of recurrent febrile convulsions could be accurately predicted in an individual child. The prediction seemed especially inaccurate in children belonging to the intermediate group. Moreover, genetic ${ }^{10-12}$ and probably socioeconomic factors may influence the type, number, interplay, and power of the predictors, and the items used to assess the recurrence risk in our study may not be appropriate for all geographical locations. Biased reporting of the family history and the type of convulsion may further reduce the predictive value.

Most studies, including ours, agree that early age at the time of the first febrile seizure is the most powerful predictor for subsequent febrile convulsions. ${ }^{1-4}{ }^{10}$ In the perinatal collaborative study only two risk factors were identified-early age at the time of initial seizure and a history of epilepsy in the near family, whereas a complex first febrile seizure and sex were not related to the recurrence rate. ${ }^{413}$ Van den Berg did not find any other characteristics of the initial seizure related to recurrence rate. ${ }^{2}$ Wallace found that an initial complex seizure, a family history of epilepsy, and social class were significantly associated with high recurrence rate. ${ }^{3}{ }^{14}$ The selection of sex as a risk factor ${ }^{1012}$ may be related to a stratification bias and could not be confirmed in the collaborative or the present study.

Our recent study indicated that short term diazepam prophylaxis reduced the 18 month recurrence rate from $39 \%$ to $12 \%$ and thus prevented two thirds of all further febrile fits. ${ }^{6}$ Stratification showed a remarkably wide range of recurrence rates in untreated children, a uniformly low recurrence rate in response to diazepam prophylaxis at times of fever, and an appreciable difference in the efficacy of prophylaxis, in terms of risk reduction.

The National Institutes of Health consensus recommended that long term prophylaxis by phenobarbitone or valproic acid should be reserved for high risk children, that is a small group with a high risk of developing epilepsy. ${ }^{15}$ By giving a few doses of diazepam per year, however (an average of five in the Copenhagen area), effective seizure control and emotional stability in the family can be obtained $^{6}$ without the many risks associated with long term prophylaxis. ${ }^{16-18}$ No serious side effects were observed from diazepam prophylaxis. In the prophylaxis group, the parents described mild, transient side effects in $65 \%$ : sedation $(36 \%)$, euphoria $(15 \%)$, ataxia $(8 \%)$, aggressiveness $(2 \%)$. One child $(0.6 \%)$ had a dental fracture probably related to ataxia. ${ }^{6}$ The parents managed the treatment surprisingly well, and compliance problems were minimal, provided that careful instruction concerning administration procedures and side effects were given. Only $4 \%$ of the children in the prophylaxis group had a recurrent febrile seizure despite alleged adequate prophylaxis. In only $4 \%$ of children allocated to prophylaxis a recurrence of febrile convulsions occurred because administration of diazepam was deliberately omitted by the parents.

The present study shows that the main effect of diazepam prophylaxis at times of fever is to provide effective seizure control in high and intermediate risk children, but that it is ineffective in the lowest risk group, comprising almost one third of the children. Our findings favour a selective prophylaxis policy, ${ }^{15} 19$ and the candidates for prophylaxis may be identified by means of the risk assessment model, based on factors readily obtainable in most children.

Supported by grants 12-2922 and 12-3674 from the Danish Medical Research Council.

I thank Svend Larsen, Herlev University Hospital, for performing the data processing and statistical analysis.

\section{References}

1 Frantzen E, Lennox-Buchthal MA, Nygaard A. Longitudinal EEG and clinical study of children with febrile convulsions. Electroencephalogr Clin Neurophysiol 1968;24:197.

2 Van den Berg BJ. Studies of convulsive disorders in young children. III. Epilepsia 1974;15:177-90.

3 Wallace SJ. Recurrence of febrile convulsions. Arch Dis Child 1974;49:763-75.

4 Nelson KB, Ellenberg JH. Predictors of epilepsy in children who have experienced febrile seizures. $N$ Engl $J \mathrm{Med}$ 1976;295:1029-33.

5 Knudsen FU. Successful intermittent diazepam prophylaxis in febrile convulsions: preliminary results of a prospective, controlled study. In: Dam M, Gram L, Penry K, eds. Advances in epileptology: XIIth epilepsy international symposium. New York: Raven Press, 1981;165-8. 
6 Knudsen FU. Effective short-term diazepam prophylaxis in febrile convulsions. $J$ Pediatr 1985; 106:487-90.

${ }^{7}$ Knudsen FU. Plasma-diazepam in infants after rectal administration in solution and by suppository. Acta Paediatr Scand 1977;66:563-7.

${ }^{8}$ Knudsen FU, Vestermark S. Prophylactic diazepam or phenobarbitone in febrile convulsions: a prospective, controlled study. Arch Dis Child 1978;53:660-3.

9 Cox DR. Regression models and life tables. J R Stat Soc B 1972;34:187-220.

10 Lennox-Buchthal MA. Febrile convulsions: a reappraisal. Electroencephalogr Clin Neurophysiol 1973;Suppl 32.

1 Ueoka K, Nagano H, Kumanomidou U, Kobuchi S, Kagitani T. Clinical and electroencephalographic study in febrile convulsions with special reference to follow-up study. Brain Dev 1979;1:196.

12 Thorn I. Prevention of recurrent febrile seizures: intermittent prophylaxis with diazepam compared with continuous treatment with phenobarbital. In: Nelson KB, Ellenberg JH, eds. Febrile seizures. New York: Raven Press 1981:119-26.

${ }^{13}$ Nelson KB, Ellenberg JH. Prognosis in children with febrile seizures. Pediatrics 1978;61:720-7.
14 Wallace SJ, Smith JA. Recurrence of convulsions in febrile children on no anticonvulsant. In: Dam M, Gram L, Penry K, eds. Advances in epileptology: XIIth epilepsy international symposium. New York: Raven Press, 1981:499-504.

15 Nelson KB, Ellenberg KH, (eds). Consensus statement on febrile seizures. In: Febrile seizures. New York: Raven Press, 1981:301-6.

16 Wolf SM, Forsythe A. Behavior disturbances, phenobarbital, and febrile seizures. Pediatrics 1978;61:728-31.

17 Camfield CS, Camfield PR. Side effects of phenobarbital in toddlers: behavioral and cognitive aspects. $J$ Pediatr 1979;95:361-5.

${ }^{18}$ Wilson JT. Observed and potential risks of anticonvulsant medications in children. In: Nelson KB, Ellenberg JH, eds. Febrile seizures. New York: Raven Press, 1981:153-68.

19 Addy DP. Prophylaxis and febrile convulsions. Arch Dis Child 1981;56:81-3

Correspondence to Dr F U Knudsen, Department of Paediatrics, Glostrup Hospital, DK 2600 Glostrup, Denmark.

Received 11 July 1985 